\title{
Brain natriuretic peptide is more than a marker
}

\author{
$B N P$ is likely to change the way we screen for, investigate and treat heart failure
}

The emergence of natriuretic peptides as significant factors in cardiac haemodynamic function has kindled much interest among clinicians and researchers. Of the three major natriuretic peptides viz. atrial natriuretic peptide (ANP) synthesised in the atria, brain natriuretic peptide (BNP) synthesised in the heart's ventricles, and C-type natriuretic peptide (CNP) synthesised in the brain, BNP has stood out as the hormone with greatest promise. The value of BNP both as a diagnostic and prognostic marker of cardiac dysfunction and as a potentially useful therapeutic agent in heart failure are exciting prospects.

BNP was first isolated from porcine brain and later from porcine, rat and human hearts, predominantly from the ventricles (1). The main trigger for BNP secretion is myocardial stretch from ventricular filling pressure and abnormal ventricular wall tension (2). The blood levels of BNP show good correlation with accepted indices of ventricular systolic dysfunction, and also reflect pressure overload in left ventricular hypertrophy as in hypertension and hypertrophic cardiomyopathy $(3,4)$. BNP released from storage granules in the myocardium binds to membrane receptors, and through increased production of cGMP, which acts as a second messenger, stimulates the nitric oxide system. Because of its vasodilator and natriuretic actions BNP serves as an excellent foil to counteract the effects of the renin-angiotensin-aldosterone and sympathetic systems that are activated in heart failure.

The most widely investigated use of BNP is in heart failure. Heart failure is a common condition with a prognosis worse than that of most cancers. Accurate and early diagnosis and treatment of heart failure improve mortality and morbidity. The clinical diagnosis of heart failure is usually reinforced by electrocardiography, chest radiography and echocardiography. These methods lack sensitivity and specificity, particularly in indentifying mild to moderate heart failure (5). Measurement of BNP is a highly sensitive and moderately specific method of diagnosing heart failure (6). It is superior to other neuroendocrine markers as an indicator of the severity of heart failure as judged by the usual indices of left ventricular dysfunction (7). In addition, BNP estimation could help to predict cardiac events in patients with heart failure and other cardiovascular diseases such as acute coronary syndromes (8). However, the assessment of BNP cannot reliably distinguish between systolic dysfunction and diastolic dysfunction.

The usefulness of measuring BNP in assisting in the diagnosis of patients with dyspnoea is by distinguishing a cardiac from a pulmonary cause (9). Blood levels of BNP are clearly elevated in heart failure and help to distinguish it from respiratory conditions such as chronic obstructive airways disease where levels are normal.

Apart from aiding the diagnosis of ventricular dysfunction, BNP is an important prognostic marker. In community studies BNP appears to be a powerful predictor of death in middle-aged and elderly populations. In one study it was a better predictor of death than the level of left ventricular dysfunction in an unselected population (10). BNP's superior predictive value is that it may reflect a summation of risk factors that affect mortality such as renal impairment, age, and left ventricular hypertrophy and left ventricular dysfunction.

In acute myocardial infarction BNP has been shown in several studies to be a strong and independent predictor of survival $(11,12)$. BNP appears to be a better marker than catecholamine and ANP in predicting outcome after acute myocardial infarction. Estimation of BNP helps to improve risk stratification and identify patients for closer follow up and aggressive intervention.

BNP levels are closely related to left ventricular remodelling, an important determinant of outcome after myocardial infarction (13). The falling levels of BNP with improvement of heart failure indicates that BNP levels may be used to guide the treatment. A study from Glasgow suggests that titration of angiotensin converting enzyme inhibitor treatment using serial BNP measurements leads to a greater inhibition of the renin-angiotensin-aldosterone system and a significant fall in heart rate compared with empirical treatment (14).

The results of a study that screened a Japanese population by measuring BNP has emphasised the versatility of this hormone in screening for cardiac abnormalities beyond the diagnosis of left ventricular dysfunction (15). In subjects who were free of heart disease, over $95 \%$ showed plasma BNP concentrations of under $50 \mathrm{pg} / \mathrm{ml}$. In those with some form of heart disease, about $90 \%$ had levels over $50 \mathrm{pg} / \mathrm{ml}$. The plasma BNP test had a sensitivity of $100 \%$ for previous myocardial infarction, lone atrial fibrillation or flutter, hypertensive heart disease and valvular heart disease.

BNP measurement is unlikely to replace well established procedures to assess cardiac function but may allow the more judicious use of invasive, costly and time consuming investigations. The main value of measuring plasma BNP in those with suspected heart disease is that a normal value indicates a low risk of cardiac impairment. A clear case is emerging that measurement of BNP can be used to identify patients requiring cardiac investigations such as echocardiography leading to a more cost effective and efficient way of allocating scarce resources. However, more information is needed to identify the normal values for different populations and divergent patient groups.

Plasma BNP level may be useful marker of left ventricular hypertrophy in hypertension. It rises progressively with increasing severity of hypertension, particularly when ventricular hypertrophy is present. This presumably reflects increases in ventricular muscle mass associated with increased synthesis and secretion of BNP from ventricular tissue (16). The elevated levels are not seen at the stage of compensatory left ventricular hypertrophy, but only when it is advanced and associated with fibrosis (17).

Elevated levels have been identified in a number of other situations that have potential clinical use. Early identification of cardiotoxicity caused by drugs is one 
instance where it may have a role in monitoring therapy but this require further evaluation (18).

The availability of simple rapid assays which are both accurate and reliable, and do not involve complex procedures for handling samples will change the future management of patients presenting with cardiovascular symptoms. One such assay of BNP suitable for bedside testing requires six drops of blood, and the results will be available in 15 minutes. This has been approved by the Food and Drug Administration of the USA for use in heart failure (19). It is important to diagnose heart failure early, and the availability of new assays of BNP will contribute to accurate and speedy diagnosis. The elderly without heart failure have higher levels than younger people, and this should be taken into consideration when interpreting the result (20). At present BNP testing is only recommended for use along the guidelines developed by the European Society of Cardiology Task Force but it will be included in the revised guidelines of other organisations (21).

The favourable haemodynamic properties of BNP make it an attractive therapeutic agent in heart failure. Nesiritide, a recombinant human B-type natriuretic peptide, appears to be superior to intravenous nitroglycerine in improving short term haemodynamic function in decompensated heart failure (22). Its widespread use will be limited until ways are found to extend its action by overcoming breakdown by endopeptidases.

Estimation of BNP offers an exciting non-invasive criterion for screening, diagnosis and risk stratification in serious cardiac disorders. If the initial robustness of this marker is confirmed in future studies in different populations, BNP seems all set to emerge as a powerful diagnostic tool in our day-to-day assessment of patients with cardiovascular disease. Its therapeutic potential is still in the early stages of development, and as its pathophysiological mechanisms get more clearly defined, BNP will certainly become much more than a mere marker.

\section{References}

1. Sudoh T, Kangawa K, Minamino N, Matsuo H. A new natriuretic peptide in porcine brain. Nature 1988; 332: 78-81.

2. Kinnunen P, Vuolteenano O, Ruskoaho H. Mechanisms of atrial and brain natriuretic peptide release from rat ventricular myocardium: effect of stretching. Endocrinology 1993; 132: 1961-70.

3. Kohno M, Hono T, Yokokawa K, Murakawa K, Yasunari K, Akioka $\mathrm{K}$, et al. Brain natriuretic peptide as a cardiac hormone in essential hypertension. American Joumal of Medicine 1992; 92: 29-34.

4. Hasegawa K, Fugiwara H, Doyama K, Miyamae M, Fujiwara T, Suga $\mathrm{S}$, et al. Ventricular expression of brain natriuretic peptide in hypertrophic cardiomyopathy. Circulation 1993; 88: 372-80.

5. Clarke KW, Gray D, Hampton JR. Evidence of inadequate investigation and treatment of patients with heart failure. British Heart Journal 1994; 72: 584-7.

6. Bettencourt P, Ferreira A, Dias P, Castro A, Martins L, CerqueiraGomes M. Evaluation of brain natriuretic peptide in the diagnosis of heart failure. Cardiology 2000; 93:19-25.
7. Groenning BA, Nilsson JC, Sondergaard L, Kjaer A, Larsson HB, Hildebrandt PR. Evaluation of impaired left ventricular ejection fraction and increased dimensions by multiple neurohumoral plasma concentrations. European Journal of Heart Failure 2001; 3: 699-708.

8. Cowie MR. BNP: soon to become a routine measure in the care of patients with heart failure? Heart 2000; 83: 617-8.

9. Morrison LK, Harrison A, Krishnaswamy P, Kazanegra R, Clopton P, Maisel A. Utility of a rapid B-natriuretic peptide assay in differentiating congestive heart failure from lung disease in patients presenting with dyspnoea. Journal of the American College of Cardiology 2002; 39: 202-9.

10. MeDonagh TA, Cunningham DA, Morrison CE, MeMurray JJV, Ford I, Morton JJ, et al. Left ventricular dysfunction, natriuretic peptides and mortality in an urban population. Heart 2001; 86: 21-6.

11. Omland T, Aakvaag A, Bonarjee VVS, et al. Plasma brain natriuretic peptide as an indicator of left ventricular function and long term survival after myocardial infarction. Circulation 1996; 93: $1963-9$.

12. Arakawa N, Nakamura M, Aoki H, et al. Plasma brain natriuretic peptide concentration predicts survival after acute myocardial infarction. Journal of the American College of Cardiology 1996; 27: 1656-61.

13. Crilley JG, Farrer M. Left ventricular remodelling and brain natriuretic peptide after first myocardial infarction. Heart 2001; 86: $638-42$.

14. Murdoch DR, McDonagh T, Byrne J, et al. Titration of vasodilator therapy in chronic heart failure according to plasma brain natriuretic peptide concentration: randomised comparison of hemodynamic and neuroendocrine effects of tailored versus empirical therapy. American Heart Journal 1999; 138: 1126-32.

15. Nakamura M, Endo H, Nasu M, Arakawa N, Segawa T, Hiramori $\mathrm{K}$. Value of plasma B-type natriuretic peptide measurement for heart disease screening in a Japanese population. Heart 2002; 87:131-5.

16. Takeda T, Kohno M. Brain natriuretic peptide in hypertension. Hypertension Research 1995; 18: 259-66.

17. Sakata Y, Yamamoto K, Masuyama T, Mano T, Nishikawa N, Kuzuya T, Miwa T, Hori M. Ventricular production of natriuretic peptides and ventricular structural remodelling in hypertensive heart failure. Journal of Hypertension 2001; 19: 1905-12.

18. Nousiainen T, Vanninen E, Jantunen E, Puustinen J, Remes J, Rantala A, et al. Natriuretic peptides during the development of doxorubicin-induced left ventricular diastolic dysfunction. Journal of Internal Medicine 2002; 251: 228-34.

19. Peacock WF. The B-type natriuretic peptide assay; a rapid test for heart failure. Cleveland Clinical Journal of Medicine 2002; 69: 243-51.

20. Nageh T, Chin D, Cooke JC, Meehan N, Monaghan MJ, Sherwood RA. Interpretation of plasma brain natriuretic peptide concentrations may require adjustment for patient's age. Annals of Clinical Biochemistry 2002; 39: 151-3.

21. Task Force of the European Society of Cardiology. Guidelines for the diagnosis and treatment of chronic heart failure. European Heart Joumal 2001; 22: 1527-60.

22. Intravenous nesiritide vs nitroglycerin for treatment of decompensated congestive heart failure: a randomized controlled trial. Journal of the American Medical Association 2002; 287: 1531-40. 